\title{
Echocardiography-guided aortic cannulation by the Seldinger technique for type A dissection with cerebral malperfusion
}

Shinichiro Shimura, MD, PhD, ${ }^{\mathrm{a}}$ Shigeto Odagiri, MD, ${ }^{\mathrm{a}}$ Hidekazu Furuya, MD, ${ }^{\mathrm{b}}$ Kimiaki Okada, MD, ${ }^{\mathrm{a}}$ Keisuke Ozawa, MD, ${ }^{a}$ Haruhiro Nagase, $\mathrm{MD},{ }^{a}$ Masaomi Yamaguchi, MD, ${ }^{\mathrm{b}}$ and Yasunori Cho, MD, $\mathrm{PhD}^{\mathrm{a}}$

\section{ABSTRACT}

Objective: The purpose of this study was to assess the efficacy of echocardiographyguided ascending aortic central cannulation using the Seldinger technique during surgery for type A acute aortic dissection complicated by stroke due to cerebral malperfusion.

Methods: Between April 2007 and December 2017, 208 patients with type A acute aortic dissection underwent echocardiography-guided ascending aortic central cannulation using the Seldinger technique. We analyzed 16 of these patients $(7.7 \%$; median age, 63 years; 8 men) with stroke due to cerebral malperfusion, including 10 in a comatose state (Glasgow Coma Scale $\leq 8$ ) and 6 with hemiplegia (manual muscle test $\leq 1$ ) on hospital arrival. The Modified Rankin Scale was used to evaluate activities of daily living.

Results: The median time from onset of symptoms to establishment of cardiopulmonary bypass was 327 (176-561) minutes. The median time from the start of surgery to establishment of cardiopulmonary bypass was 34 (30-44) minutes. The mortality rate was $6.3 \%(1 / 16)$. In patients with preoperative coma, the Glasgow Coma Scale improved significantly after surgery from 4.5 to 15 at 30 days $(P<.001)$. In patients with preoperative hemiplegia, 4 showed improved motor function on the manual muscle test score at 90 days. In all patients, Modified Rankin Scale scores improved significantly from 5.0 preoperatively to 1.0 after follow-up $(P<.001)$.

Conclusions: Echocardiography-guided ascending aortic central cannulation using the Seldinger technique has potential as a rapid and reliable perfusion route during surgery for type A acute aortic dissection complicated by stroke due to cerebral malperfusion. (J Thorac Cardiovasc Surg 2020;159:784-93)

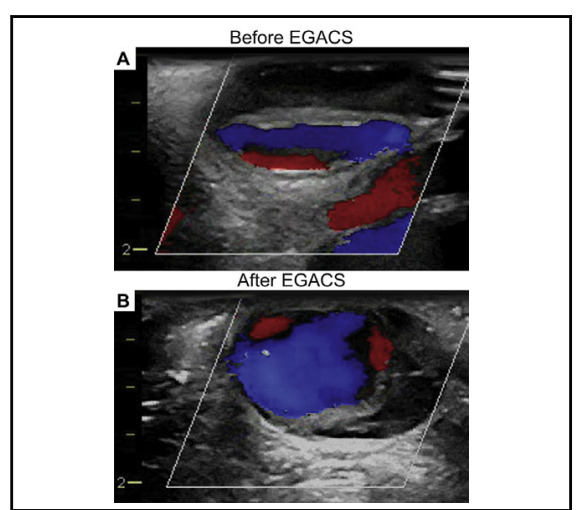

Doppler echocardiography of the BCA (A) before and (B) after EGACS.

\section{Central Message}

EGACS provides rapid antegrade perfusion that has the potential to facilitate immediate central aortic repair for TAAAD complicated by $\mathrm{CM}$.

\section{Perspective}

In this study, we used EGACS as a supportive technique during surgery for TAAAD complicated by CM. To our knowledge, this is the first study to evaluate the efficacy of this perfusion strategy in relieving $\mathrm{CM}$ associated with dynamic obstruction in TAAAD.

See Commentaries on pages 794 and 796.
From the ${ }^{a}$ Department of Cardiovascular Surgery, School of Medicine, Tokai University, Kanagawa, Japan; and ${ }^{\mathrm{b}}$ Department of Cardiovascular Surgery, Tokai University Hachioji Hospital, Tokyo, Japan.

This manuscript was presented at: The American Association for Thoracic Surgery Aortic Symposium as Director's Choice, New York, New York, April 26-27, 2018. Date and number of Institutional Review Board approval: June 22, 2018; 18R-075.

Received for publication Nov 11, 2018; revisions received Feb 6, 2019; accepted for publication Feb 18, 2019; available ahead of print April 18, 2019.

Address for reprints: Shinichiro Shimura, MD, PhD, Department of Cardiovascular Surgery, School of Medicine, Tokai University, 143 Shimokasuya, Isehara, Kanagawa 259-1193, Japan (E-mail: shimura@is.icc.u-tokai.ac.jp).

$0022-5223 / \$ 36.00$

Copyright (c) 2019 by The American Association for Thoracic Surgery

https://doi.org/10.1016/j.jtcvs.2019.02.097
According to the International Registry of Acute Aortic Dissection study, ${ }^{1}$ type A acute aortic dissection (TAAAD) with cerebral malperfusion (CM) has poor outcomes, and $6 \%$ of TAAAD cases are complicated by stroke, resulting in higher in-hospital mortality or a longer stay. On the

- Scanning this $\mathrm{QR}$ code will take you to the article title page to access supplementary information. 


$$
\begin{aligned}
& \text { Abbreviations and Acronyms } \\
& \text { AXA }=\text { axillary artery } \\
& \mathrm{BCA}=\text { brachiocephalic artery } \\
& \mathrm{CCA}=\text { common carotid artery } \\
& \mathrm{CM}=\text { cerebral malperfusion } \\
& \mathrm{CPB}=\text { cardiopulmonary bypass } \\
& \mathrm{CT}=\text { computed tomography } \\
& \mathrm{EGACS}=\text { echocardiography-guided ascending } \\
& \text { aortic central cannulation using the } \\
& \text { Seldinger technique } \\
& \mathrm{FA}=\text { femoral artery } \\
& \mathrm{GCS}=\text { Glasgow Coma Scale } \\
& \mathrm{IQR}=\text { interquartile range } \\
& \mathrm{MMT}=\text { manual muscle test } \\
& \mathrm{mRS}=\text { Modified Rankin Scale } \\
& \mathrm{POD}=\text { postoperative day } \\
& \mathrm{rSO}=\text { regional oxygen saturation } \\
& \mathrm{TAAAD}=\text { type A acute aortic dissection } \\
& \mathrm{TAR}=\text { total arch replacement }
\end{aligned}
$$

other hand, several studies ${ }^{2-5}$ have confirmed the efficacy of early surgery for TAAAD complicated by CM. We use echocardiography-guided ascending aortic central cannulation using the Seldinger technique (EGACS) as a supportive technique during surgery for TAAAD. The EGACS technique is simple, and central cannulation can be achieved using the Seldinger technique ${ }^{6}$ because it promptly provides antegrade blood flow in the true lumen coupled with core cooling. This mechanism might be effective for correction of $\mathrm{CM}$, especially in cases associated with dynamic obstruction; however, there is no evidence of this. This study aimed to evaluate the outcomes of EGACS for TAAAD complicated by stroke due to $\mathrm{CM}$.

\section{MATERIALS AND METHODS \\ Patients}

From April 2007 to December 2017, 229 consecutive patients with TAAAD underwent graft replacement and 208 patients were treated with EGACS. The remaining 21 patients with ascending aortic rupture or a false lumen with thrombosis encircling the ascending aorta who were treated with single cannulation from the femoral artery (FA) were excluded from this study. To exclude coma caused by shock and circulatory failure, patients in a coma but without dissection in branches of the aortic arch were excluded from this study. During the same period, 5 patients who were not able to undergo surgery because of deep coma with brain death (1 patient), coma with cardiopulmonary arrest after arrival at the hospital ( 2 patients), and rejection of surgery for being bedridden or for religious reasons ( 2 patients) were excluded from this study.

The study participants consisted of $16(7.7 \%)$ of the 208 patients treated for TAAAD complicated by stroke due to CM using EGACS (Video 1) on arrival at the hospital. Median patient age was 63 years (range, 32-83), and 8 were male. The patients' backgrounds are summarized in Table 1. Our hospital is a core hospital in the western district of Kanagawa Prefecture, Japan, and initial clinical diagnosis was performed in the advanced emergency center of our hospital. This single-center retrospective study was

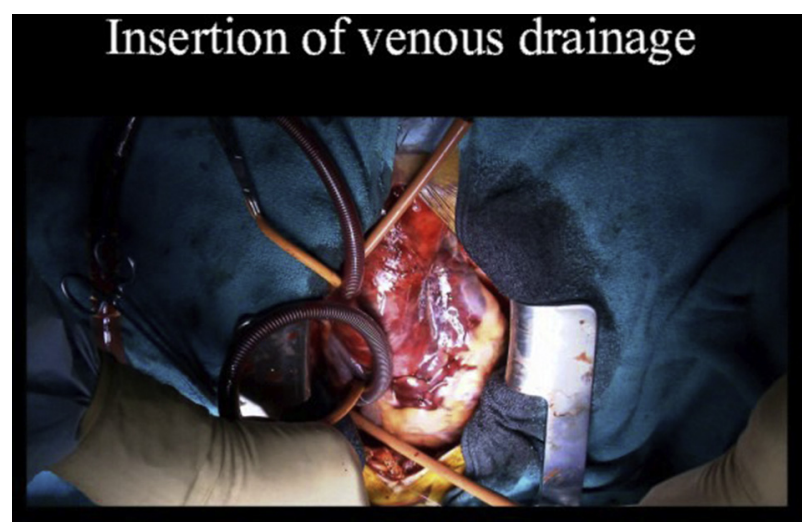

VIDEO 1. A general method of EGACS and a representative case of a 68-year-old patient with left hemiplegia and in a comatose state (GCS 7) on hospital arrival who underwent EGACS. EGACS, Echocardiographyguided ascending aortic central cannulation using the Seldinger technique; GCS, Glasgow Coma Scale. Video available at: https://www.jtcvs.org/ article/S0022-5223(19)30565-3/fulltext.

performed using data obtained from medical records. All patients or relatives gave informed consent. The study was approved by the institution's ethics committee.

\section{Neurologic Evaluation}

Neurologic evaluation on arrival at the hospital was performed by an emergency physician or neurologist. Coma and hemiplegia were defined as neurologic deficits. The level of impaired consciousness was assessed using the Glasgow Coma Scale (GCS), ${ }^{8}$ and GCS 8 or less on arrival was defined as preoperative coma. Hemiplegia was assessed using the manual muscle test (MMT), ${ }^{9}$ and MMT 1 or less was defined as hemiplegia on arrival. To exclude the uncertainty in false-positive neurologic findings, GCS 8 or less and MMT 1 or less were defined as neurologic deficits. Transient impaired consciousness was excluded. Although all patients in this study underwent preoperative head computed tomography (CT) due to the existence of prehospital neurologic impairment, imaging of the brain, such as CT or magnetic resonance imaging, was not routinely conducted to allow performance of surgery as promptly as possible. Ten patients presented in a comatose state, and 6 patients presented with hemiplegia in a semicomatose state or not. Patients with transient consciousness disturbance were not included in this study. Before and after surgery and during follow-up, the level of consciousness of patients with preoperative coma was assessed using the GCS, and the motor function of the paralytic side was evaluated using MMT in patients with preoperative hemiplegia, performed strictly according to the common manual of GCS and MMT. The Modified Rankin Scale (mRS) was used to evaluate the degree of independence in activities of daily living during follow-up.

\section{Surgical Procedure}

Patients were placed in the standard supine position. Cerebral blood flow was monitored by echoencephalography to confirm blood flow in the true lumen in a branch of the aortic arch $(\mathrm{n}=16)$. Near-infrared spectroscopy was used to measure regional oxygen saturation $\left(\mathrm{rSO}_{2}\right)$ in the bilateral frontal regions using a noninvasive monitoring system (INVOS 5100C, Medtronic, Boulder, Colo $(n=9)$. Transesophageal echocardiography was used to visualize the extent of aortic dissection during the procedure. Surgery was performed via a median sternotomy. Extracorporeal circulation was achieved by venous drainage from the superior and inferior vena cava, with both FA cannulation and EGACS in 2 patients, and EGACS alone in all other patients.

The systolic arterial pressure was controlled to less than $80 \mathrm{~mm} \mathrm{Hg}$ before EGACS. Mattress sutures were placed between the ascending aorta and pulmonary trunk using a 4-0 monofilament pledget-supported surgical 
TABLE 1. Characteristics of patients treated with ascending aortic cannulation

\begin{tabular}{lc}
\hline \multicolumn{1}{c}{ Characteristics } & No. \\
\hline No. of patients & 16 \\
Age & 63 y (range, 32-83) \\
Male/female & $8 / 8$ \\
Neurologic deficits & \\
$\quad$ Coma: GCS $\leq 8$ (with hemiparesis) & $10(3)$ \\
Hemiplegia: MMT $\leq 1$ (with semi-coma) & $6(2)$ \\
Conjugate deviation of the eyes & 8 \\
Cardiac tamponade & 5 \\
Successful cardiopulmonary resuscitation & 3 \\
History of brain attack & 1 \\
Concomitant organ malperfusion & \\
Heart (defibrillated ventricular tachycardia) & 1 \\
Spine (paraplegia) & 1 \\
Leg ischemia & 1 \\
Dissection of arch vessels & \\
BCA & 16 \\
Right CCA (with occlusion) & $12(7)$ \\
Left CCA & 7 \\
Left subclavian artery & 8 \\
Thrombosed false lumen & 3 \\
\hline TCS & \\
\hline
\end{tabular}

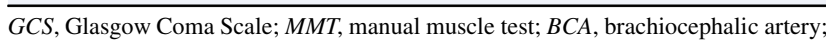
$C C A$, common carotid artery.

suture and needle. A 20F flexible spindle-shaped cannula (DLP Arterial Cannula: Medtronic Inc, Minneapolis, Minn) was inserted into the ascending aorta using the Seldinger wire technique. To install a cannula at the exact point of the true lumen, true and false channels were detected using epiaortic and transesophageal echocardiography (Vivid i Ultrasound System and i12L-RS Transducer: GE Medical Systems, Haifa, Israel; or Vivid E9 Ultrasound System and 11L-D Transducer: E Vingmed Ultrasound, Horten, Norway) to confirm the presence of the wire in the true lumen in both the ascending and descending aortas, in combination with anatomic information from preoperative CT scans.

Antegrade perfusion was started, and core cooling began at a flow rate of $2.4 \mathrm{~L} / \mathrm{min} / \mathrm{m}^{2}$. The left ventricle was vented through the right superior pulmonary vein. Carbon dioxide was continuously infused into the surgical field at a flow rate of $2 \mathrm{~L} / \mathrm{min}$. A centrifugal pump (CAPIOXR Disposable Centrifugal Pump, CX*SP45: Terumo Corp, Tokyo, Japan) was used for extracorporeal circulation. At the start of extracorporeal circulation and when a steady flow from the centrifugal pump was reached by cooling, epiaortic echocardiography was performed to confirm that the true lumen in the ascending aorta and a branch of the aortic arch was enlarged after EGACS (Figure 1). Thiopental sodium $(30 \mathrm{mg} / \mathrm{kg}$ ) was injected into the cardiopulmonary bypass (CPB) circuit just before circulatory arrest. The patients were placed in the Trendelenburg position, and circulatory arrest was established at $17.5^{\circ} \mathrm{C}$ as drained blood temperature. An arterial cannula was removed after incising the ascending aorta and confirming that the tip of the cannula was placed in the true lumen. Ascending aortic replacement was performed when an intimal tear was found in the ascending aorta or total arch replacement (TAR) was performed when it was found in the aortic arch.

After closing the false lumen with stump formation using a continuous horizontal mattress suture buttressed with Teflon felt strips through an open distal procedure, ascending aortic replacement was performed with a Dacron 1-branched graft (J Graft Shield Neo, Japan Lifeline Co, Tokyo, Japan), followed by distal anastomosis. TAR was performed with a Dacron 4-branched graft (Hemashield Platinum Plus Woven Double Velour, 4 branch, Intervascular SAS, LaCiotat, France) for branch revascularization of the aortic arch, with arch branches reconstructed first to minimize the period of circulatory arrest. We used retrograde cerebral perfusion for all patients who underwent surgery for TAAAD to flush out air and atheromatous debris and for auxiliary neuroprotection. On completion of distal repair or anastomosis of all branches of the aortic arch, retrograde cerebral perfusion was performed to flush out air and atheromatous debris within the arch vessels. It was also performed to provide cerebroprotection for 30 minutes or more after arresting the cerebral circulation. In ascending aortic replacement, after the completion of distal anastomosis, extracorporeal circulation and rewarming resumed from the branch graft at a flow rate of $2.4 \mathrm{~L} / \mathrm{min} / \mathrm{m}^{2}$. In TAR, after branch revascularization in the aortic arch, cerebral perfusion resumed from the branch graft at a perfusion volume of $10 \mathrm{~mL} / \mathrm{kg} / \mathrm{min}$. At the start of perfusion from branch grafts, $30 \mathrm{mg}$ of edaravone, ${ }^{10}$ which was approved in Japan in 2001 as a drug targeting peroxyl radicals to treat acute phase cerebral infarction, was injected via a CPB device for cerebroprotection. Before and after circulatory arrest, D-mannitol (35-45 g) was injected via the CPB device. In TAR, distal anastomosis was performed by open distal anastomosis with the elephant trunk technique while lower body circulation was arrested. Subsequently, graft-graft anastomosis was performed. During both ascending aortic graft replacement and TAR, cardioplegia was intermittently performed using antegrade selective coronary reperfusion every 30 minutes. The aortic valve was resuspended, and the proximal aortic stump was repaired as follows. Reinforcement of the dissected aortic wall using band-like felt in a false lumen with gelatin-resorcin-formalin biological glue (Caridal SA, Saint-Etienne, France) was used in the first 8 patients. In subsequent patients, base formation was performed using a double felt strip due to discontinuation of the glue. No patient simultaneously underwent coronary artery bypass graft surgery.
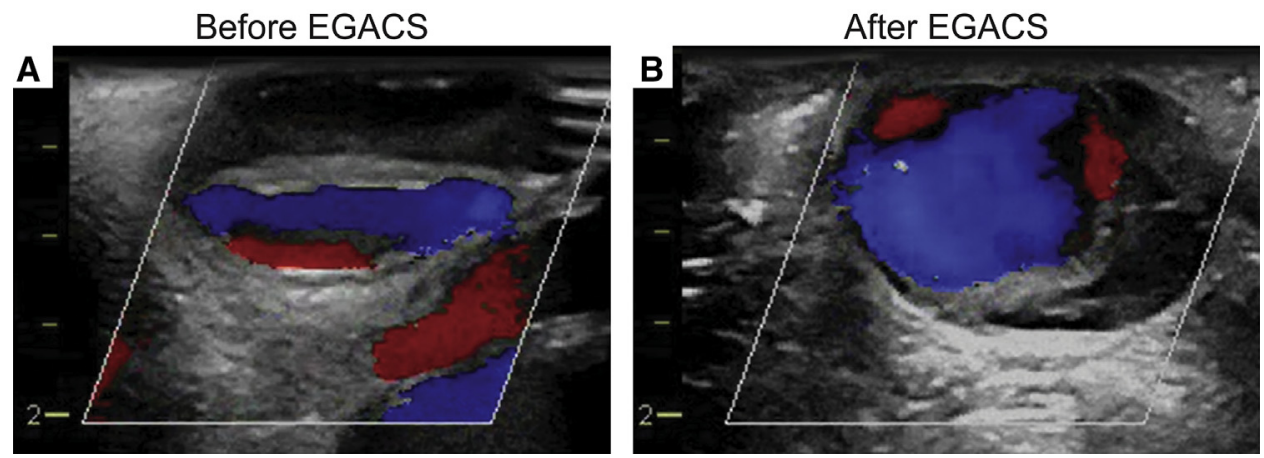

FIGURE 1. Epiaortic Doppler echocardiography shows a compressed true lumen of the BCA (A) before EGACS and enlargement of the true lumen of the BCA (B) after EGACS. EGACS, Echocardiography-guided ascending aortic central cannulation using the Seldinger technique. 
TABLE 2. Surgical variables in performance of ascending aortic cannulation

\begin{tabular}{lc}
\hline \multicolumn{1}{c}{ Variables } & Time (min) \\
\hline Onset to establishment of CPB & $327(176-561)$ \\
Start of operation to establishment of CPB & $34(30-44)$ \\
Establishment of CPB to cardiac arrest (cooling time) & $30(21-45)$ \\
CPB time & $203(128-304)$ \\
Surgery time & $378(231-917)$ \\
Brain circulatory arrest time & $33(25-45)$ \\
\hline
\end{tabular}

$C P B$, Cardiopulmonary bypass.

\section{Postoperative Neurologic Care}

All patients were examined by a neurologist. Glycerol $(200 \mathrm{~mL}$, twice/day) and edaravone ( $30 \mathrm{mg} / \mathrm{day}$ ) were routinely administered up to 14 days based on the regulations for use, until the patient was completely awake and could comply with instructions as a result of improvement of neurologic function. Rehabilitation started earlier at the bedside and mobility training started after weaning from a mechanical ventilator. Except for those with postoperative prolonged coma, patients who did not require surgical care were discharged and transferred to a rehabilitation hospital as soon as possible.

\section{Statistical Analysis}

The $\mathrm{rSO}_{2}$ in the bilateral frontal regions before and after the start of $\mathrm{CPB}$ with EGACS was evaluated by the Wilcoxon-signed rank test. Evaluations using GCS in the coma group, MMT in the hemiplegia group, and $\mathrm{mRS}$ in all patients were performed before and after surgery at different time points using the Friedman's test. The cumulative survival was estimated by the Kaplan-Meier method. All analyses were performed using SPSS v.23.0 (IBM Corp, Armonk, NY).

\section{RESULTS}

\section{Operative Findings}

Surgical data are shown in Table 2. The median time from onset to establishment of CPB was 327 (176-561) minutes. The median time from the start of surgery to establishment of CPB was 34 (30-44) minutes. The median time to cardiac arrest from establishment of CPB (cooling time) was 30 (21-45) minutes. Antegrade blood flow was obtained without breakdown of the cannulation site or cannulation to the false lumen in all patients. The median $\mathrm{rSO}_{2}$ level in the bilateral frontal regions, which was observed using

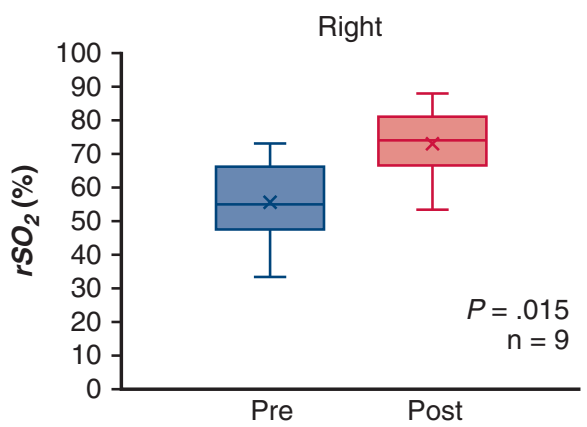

near-infrared spectroscopy, improved significantly after EGACS from $55.0 \%$ (interquartile range [IQR], $47.5 \%-65.0 \%$ ) to $74.0 \%$ (IQR, $66.5 \%-80.5 \%$ ) $(P=.015)$ on the right, and from $66.0 \%$ (IQR, $52.0 \%-72.0 \%)$ to $79.0 \%$ (IQR, $70.0 \%-84.5 \%$ ) $(P=.008)$ on the left (Figure 2). All patients underwent emergency surgery, and near-infrared spectroscopy was not performed in some cases because of limitations of staff and facilities. After incising the ascending aorta, the tip of the cannula was confirmed to be placed in the true lumen. Intimal tear was found in the ascending aorta and aortic arch in 9 and 6 patients, respectively, and was not detected in 1 patient. Ascending aortic graft replacement was performed in 10 patients and TAR in 6 patients. The time of circulatory arrest in the brain was 33 (25-45) minutes, the CPB time was 203 (128-304) minutes, and the surgery time was 378 (231-917) minutes.

\section{Early Outcomes}

One patient $(6.3 \%)$ died in the hospital. This patient had preoperative left hemiplegia associated with mildly impaired consciousness and died of severe cerebral edema on postoperative day (POD) 18. In patients in a preoperative comatose state, median postoperative GCS scores significantly improved from 4.5 (IQR, 3.8-7.3) at arrival to 11.5 (IQR, 8.8-15.0) at 7 days, 15.0 (IQR, 14.0-15.0) at 30 days, and 15.0 (IQR, 15.0-15.0) at 90 days $(P<.001)$, showing improved consciousness (Figure 3). Fourteen patients $(87.5 \%)$, including 10 with preoperative coma, had a GCS score of 15 and full recovery of consciousness at 90 days. Of the 6 patients with preoperative hemiplegia, 4 showed improved motor function on the MMT of 4 or greater at 90 days (preoperative median 0.5, IQR, 0-1.0, 30-day median 4.0, IQR, 0-4.5, and 90-day median 5.0, IQR, 2.0-5.0; $P=.057$ ), and of the remaining 2 patients, 1 was in a postoperative coma and 1 died (Figure 4). A male patient aged 36 years with preoperative hemiplegia who underwent ascending aortic graft replacement developed severe cerebral coma on POD 2 and was treated with decompressive craniectomy by neurosurgeons. Three

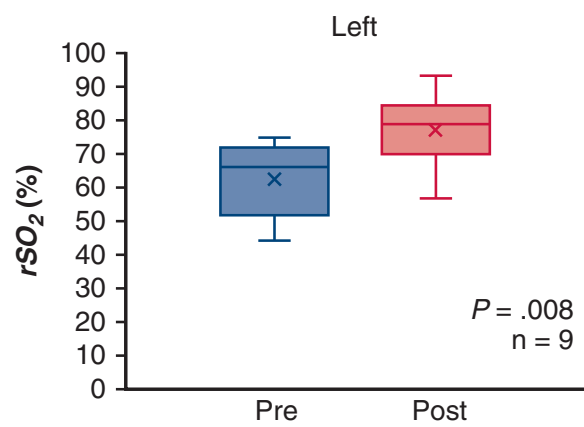

FIGURE 2. The median $\mathrm{rSO}_{2}$ levels in the bilateral frontal regions observed using near-infrared spectroscopy. Median $\mathrm{rSO}_{2}$ levels improved significantly after EGACS from $55.0 \%$ (IQR, 47.5\%-65.0\%) to 74.0\% (IQR, 66.5\%-80.5\%) $(P=.015$ ) (right) and from $66.0 \%$ (IQR, 52.0\%-72.0\%) to 79.0\% $(\mathrm{IQR}, 70.0 \%-84.5 \%)(\mathrm{P}=.008)(\mathrm{left}) . r \mathrm{SO}_{2}$, Regional oxygen saturation. 


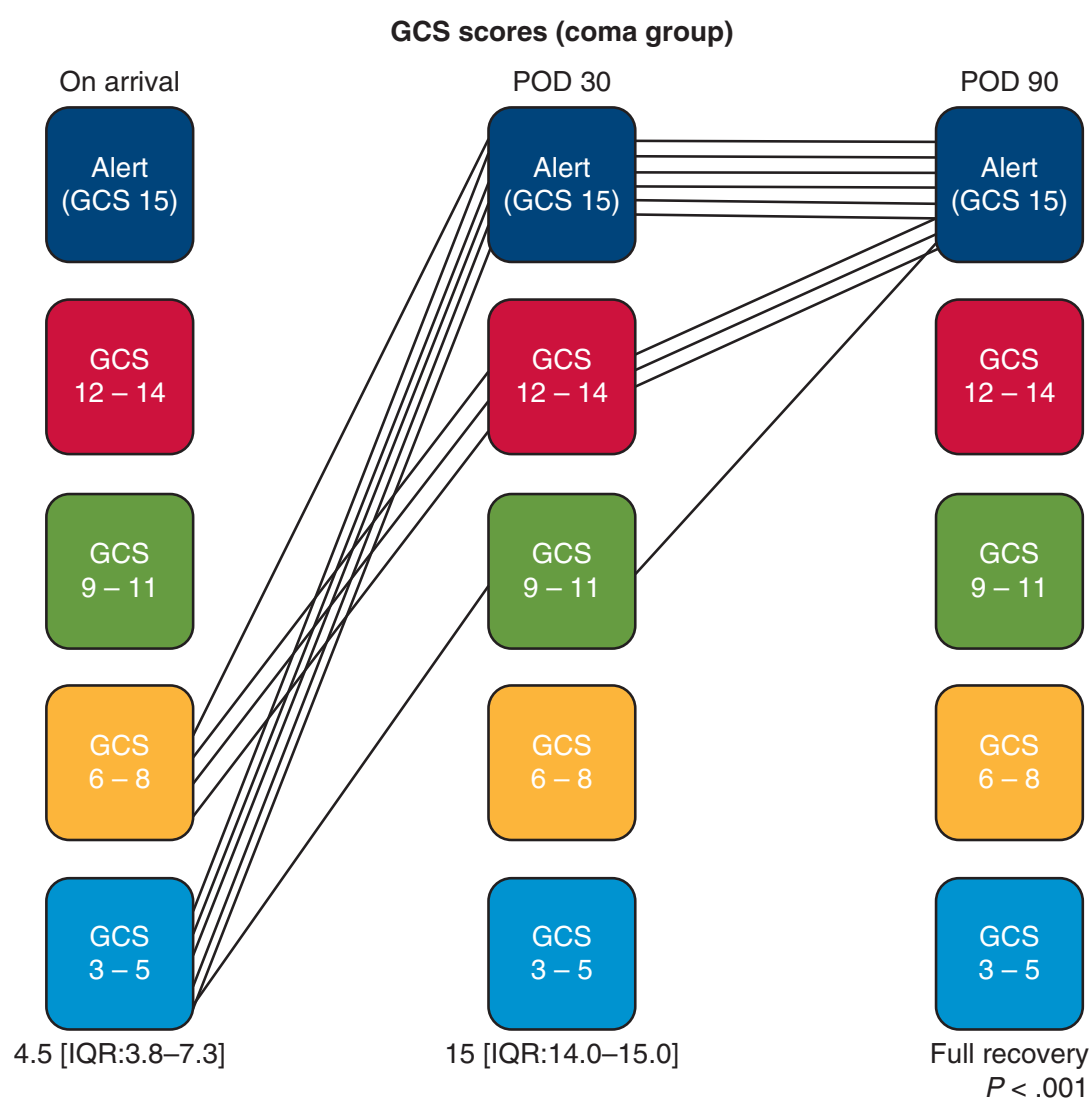

FIGURE 3. GCS scores of patients with preoperative coma. In these patients, median GCS scores improved significantly after surgery from 4.5 to 15 at 30 days, and full recovery of consciousness at 90 days was achieved. GCS, Glasgow Coma Scale; POD, postoperative day; IQR, interquartile range. This figure was made on the basis of the design of a similar figure by Tsukube and colleagues. ${ }^{3}$

patients were discharged to home, 12 patients were transferred to rehabilitation hospitals, and 1 patient was transferred to a healthcare hospital. A CT scan of a representative case with coma is shown in Figure 5.

\section{Late Outcomes}

During the final follow-up at the end of April 2018, all patients, except for 1 who died in the hospital, were alive, and 14 patients $(87 \%)$ lived at home. The mean follow-up period was $101 \pm 7$ months, and the cumulative 5-year survival was $93.8 \%$. The median $\mathrm{mRS}$ significantly improved over time; median $\mathrm{mRS}$ was 5.0 (IQR, 5.0-5.0) preoperatively, 4.0 (IQR, 3.0-4.8) at discharge, 2.0 (IQR, $0-4.8)$ at 90 days, and 1.0 (IQR, 0-4.8) at final follow-up $(P<.001)$. Independence in activities of daily living $(\mathrm{mRS} \leq 2)$ was achieved in 12 patients $(75 \%)$ during follow-up. Physical impediments remained in 3 patients, 1 with preoperative coma and paraplegia (GCS 15, mRS 5), 1 with preoperative coma and hemiplegia (GCS 15, mRS 5 ), and 1 with preoperative hemiplegia and postoperative new coma with anamnesis of decompressive craniectomy due to severe cerebral edema (GCS 5, mRS 5).

\section{DISCUSSION}

From the data in the Japan Cardiovascular Surgery Database, in 2014, the 30-day and in-hospital mortality rates after surgery for TAAAD were $8.8 \%$ and $10.6 \%$, respectively. ${ }^{11}$ Aortic branch vessel occlusion makes mortality higher in surgery for TAAAD. ${ }^{2}$ In particular, $\mathrm{CM}$ in TAAAD worsens a patient's prognosis and postoperative quality of life. ${ }^{12,13}$ Urgent surgical repair is required for TAAAD because conservative management is associated with a high incidence of early mortality. ${ }^{14,15}$ Some studies have suggested that urgent surgical repair in the presence of stroke has a risk of hemorrhagic worsening of ischemic infarction after reperfusion and before CPB and full anticoagulation. ${ }^{16,17}$ Others have suggested that delaying aortic repair until the cerebral injury stabilizes may minimize brain dysfunction, even though this exposes patients to a risk of death due to rupture. ${ }^{18}$ On the other hand, several studies ${ }^{2-5}$ have confirmed the efficacy of early surgery for TAAAD complicated by stroke. In patients with TAAAD complicated by coma who underwent emergency surgery within 5 hours after onset, Tsukube and colleagues 


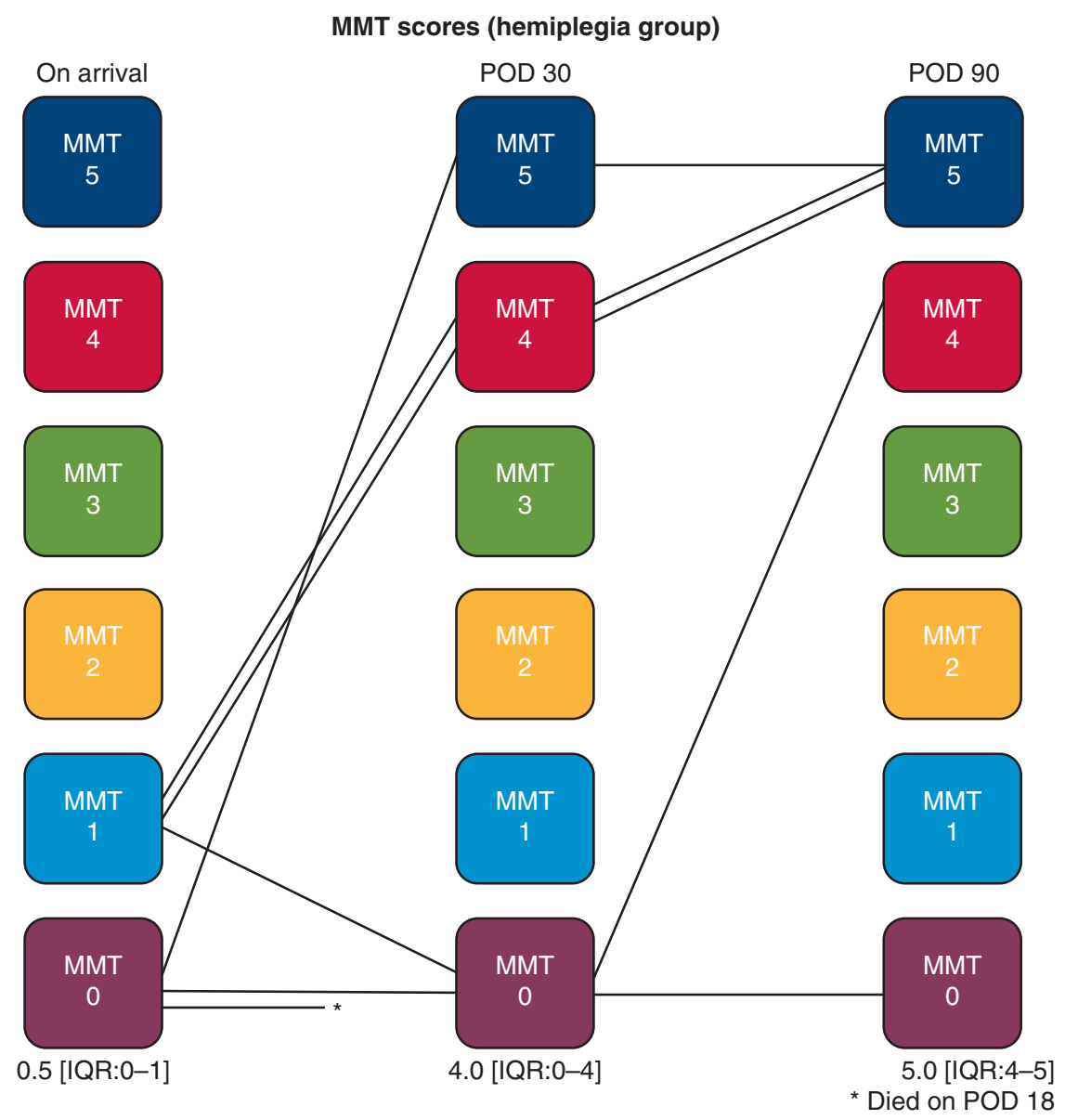

FIGURE 4. MMT scores of patients with preoperative hemiplegia. Of the 6 patients with preoperative hemiplegia, 4 showed a tendency toward improved motor function of MMT 4 or greater at 90 days. MMT, Manual muscle test; $P O D$, postoperative day; $I Q R$, interquartile range.

reported an in-hospital mortality rate of $14 \%$ and a rate of recovery of consciousness of $86 \%$. Estrera and colleagues ${ }^{4}$ reported the operative results of 16 patients with TAAAD complicated by preoperative stroke. The median time from onset of stroke to surgery was 9 hours, and $80 \%$ of patients who underwent surgical repair within 10 hours had improvement in neurologic status, whereas no patient who was operated on beyond 10 hours showed improvement. Furthermore, both the early- and long-term outcomes after immediate aortic repair for TAAAD complicated by $\mathrm{CM}$ are reported to be satisfactory. ${ }^{19}$

Ischemic penumbra is the term used to define ischemic but still viable cerebral tissue. It was classically defined as the hypoperfused tissue surrounding the ischemic core in which blood flow is too low to maintain electric activity, but sufficient to preserve ion channels. ${ }^{20}$ The 2015 guidelines of the Healthcare Professionals from the American Heart Association/American Stroke Association reported the efficacy of endovascular treatment within 8 hours of symptom onset for patients with acute ischemic stroke. ${ }^{21}$ The reduction of the time from onset to blood reperfusion to the brain is the most important parameter in surgery for TAAAD complicated by CM. In this study, all patients underwent immediate emergency surgery, with a median time from onset to establishment of CPB of 331 minutes (5 hours, 31 minutes), and the median time from the start of surgery to establishment of CPB of 34 minutes. There is a possibility that EGACS contributes to immediate blood supply within the ischemic penumbra in patients with TAAAD complicated by CM.

Although opinions on the optimal cannulation strategy for TAAAD surgery vary, ${ }^{12,22-25}$ cannulations of the FA, axillary artery (AXA), and central aorta are the major strategies for TAAAD surgery, and Abe and Usui ${ }^{22}$ indicated that each procedure has advantages and disadvantages. Orihashi ${ }^{12}$ showed that FA perfusion started promptly and was effective in patients with shock, but had a higher incidence of false lumen perfusion (4/44 cases, $9.1 \%$ ) compared with AXA perfusion, including 3 patients who developed CM. We now use EGACS only, except for patients with mesenteric malperfusion or lower-body ischemia. In cases with combined FA perfusion, we control 

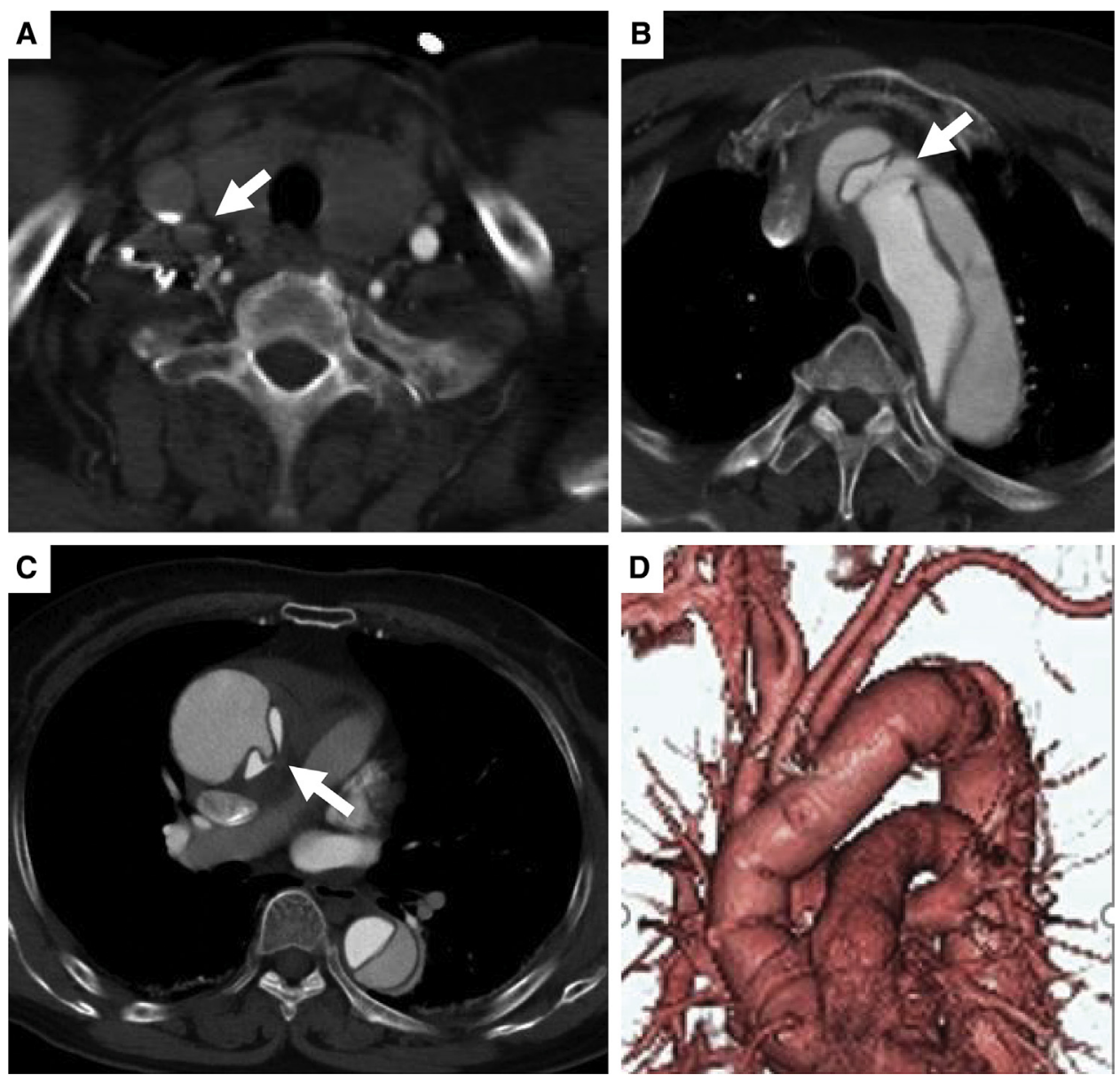

FIGURE 5. Preoperative enhanced CT scan of the thorax (A-C) and postoperative CT scan with 3-dimensional reconstruction (D) in a representative case of a 68-year-old patient with coma (GCS 7) and left hemiplegia on arrival. Total arch graft replacement was performed 3 hours after onset. The patient recovered with full consciousness and hemiplegia improved postoperatively. A, Occluded right CCA (arrow). B, Intimal tear in the aortic arch (arrow). C, Compressed true lumen of the ascending aorta (arrow). D, Postoperative CT scan with 3-dimensional reconstruction shows enlargement of true lumens of branches of the aortic arch.

the perfusion of ascending aortic flow to FA flow at a ratio of $2: 1$ using an infrared flow meter. In EGACS, the cannula is placed in the true lumen of the ascending aorta using the Seldinger wire technique under epiaortic echocardiography with confirmation of the supply site using preoperative $\mathrm{CT}^{6,7,26}$ This procedure has good outcomes, because it takes less time to establish CPB, antegrade blood flow in the true lumen is obtained, and any new CM induced by CPB might be prevented. ${ }^{6,7,27}$ Cannulation breakdown and a risk of blood supply to the false lumen are concerns. ${ }^{28,29}$ We observed $3(1.4 \% ; 3 / 208)$ cases of false lumen cannulation in early patients, 1 for each of the 3 operating surgeons. However, these cases could have been avoided with strict observance of the process described in the "Materials and Methods" section. Therefore, this has not occurred after April 2013.

The strategy of perfusion for TAAAD surgery complicated by $\mathrm{CM}$ has not been established. ${ }^{12,23}$
Although AXA perfusion is generally considered a standard cannulation method for TAAAD, Orihashi and colleagues ${ }^{30}$ reported that if dissection extends to the innominate artery and stenosis in the true lumen is complicated, the certainty of AXA perfusion is reduced. One case report showed that the thrombus in the false lumen of the right common carotid artery (CCA) was scattered to the true lumen in a patient with right CCA occlusion due to dissection. $^{31}$ Therefore, AXA perfusion for TAAAD complicated by $\mathrm{CM}$ is not completely safe. However, some studies have shown that direct cannulation of the right CCA and brachiocephalic artery (BCA) is effective. ${ }^{32,33}$ Okita and colleagues ${ }^{34}$ performed reperfusion of the right CCA before CPB in patients with TAAAD complicated by $\mathrm{CM}$, with good outcomes. This is a definite method for reperfusion on the distal side of an occluded CCA. However, to make an exact diagnosis of static or dynamic obstruction, even in patients with shock, is difficult. 

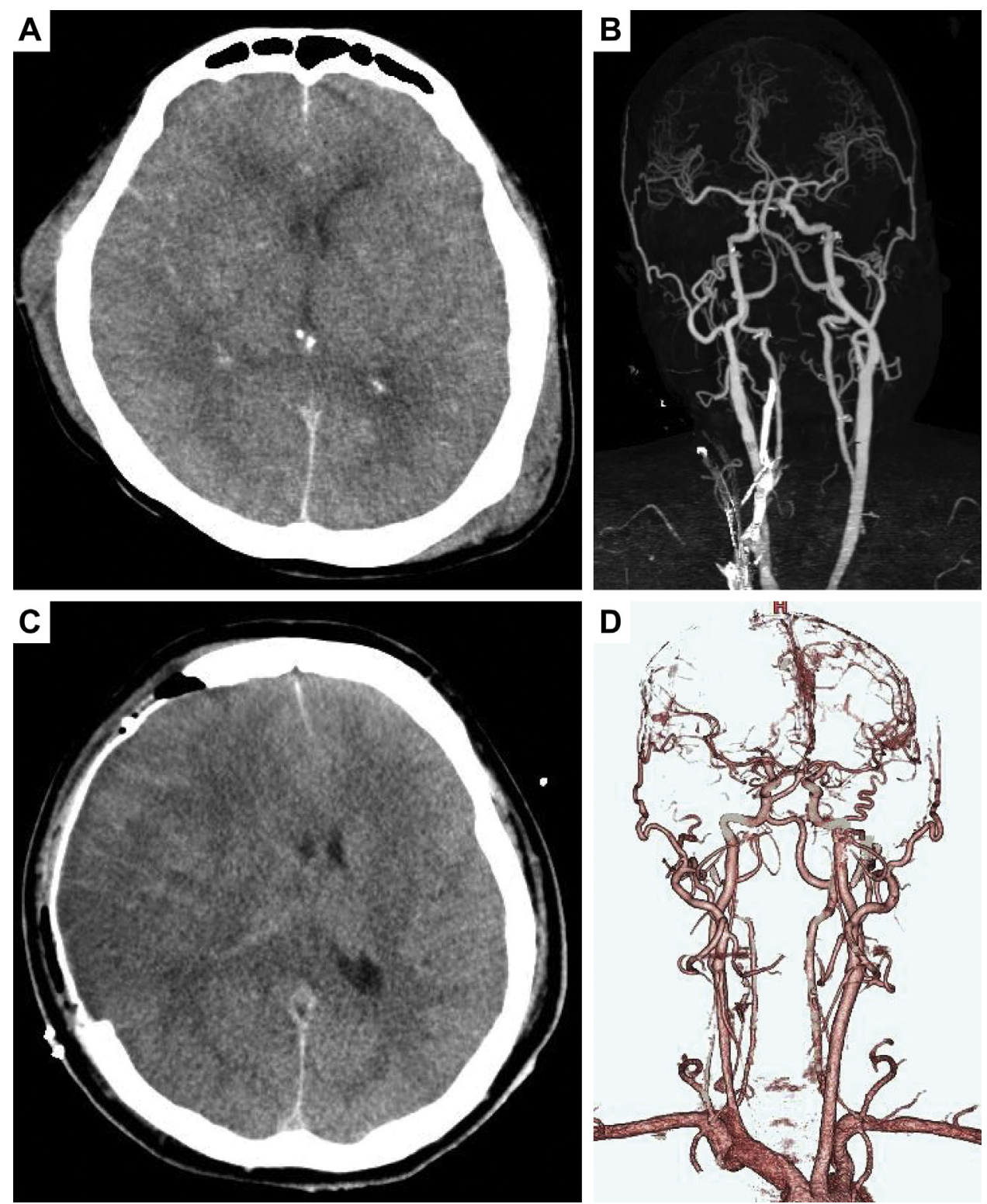

FIGURE 6. Postoperative plain CT scan of the head (A, C) and postoperative magnetic resonance imaging (B) and CT scan (D) of intracranial and cervical arteries with 3-dimensional reconstruction in patients who underwent ascending aortic graft replacement with preoperative hemiplegia without coma; 1 patient developed severe cerebral edema within 3 days of surgery and died in hospital (A, B), and 1 patient underwent decompressive craniectomy (C, D). Although the postoperative CT scans show severe cerebral edema, postoperative magnetic resonance imaging and CT scan with 3-dimensional reconstruction show patent intracranial and cervical arteries.

Therefore, it is preferable to promptly perform EGACS for true lumen perfusion and cooling in patients with stenosis in the true lumen due to dynamic obstruction, and add direct cannulation of the CCA and BCA if required.

The intraoperative cerebroprotection used in this study was deep hypothermic circulatory arrest. We consider that cannulation of dissected branches of the aortic arch carries a risk of development of new intimal tears in the aortic arch. Cerebroprotection is the highest priority for critical patients with TAAAD with $\mathrm{CM}$; therefore, we consider that a combination of quick and simple EGACS and deep hypothermic circulatory arrest is appropriate.

Intimal tear in the distal site of the aortic arch, distant from the cannulation site, was found in 7 patients in this study. However, no new malperfusion on the distal side of the cannulated site was found in any of the 16 patients. Heart beats decreased as central cooling time increased, and an enlarged true lumen of BCA was seen on intraoperative echocardiography, as shown in Figure 1. Inoue and colleagues ${ }^{7}$ showed that ascending aortic 
perfusion improved dynamic obstruction in the true lumen caused by increased pressure in the false lumen, along with prompt core cooling by antegrade blood flow in the true lumen. In addition, a centrifugal pump is always used for CPB in our hospital; therefore, new malperfusion was unlikely because the above mechanism was likely controlled by the pump-assisted, nonpulsating steady flow. The result that the median $\mathrm{rSO}_{2}$ level in the bilateral frontal regions improved significantly after EGACS supports the facts mentioned.

Two patients who underwent ascending aortic graft replacement with preoperative hemiplegia without coma developed severe cerebral edema within 3 days of surgery. One died on POD 18; the other patient underwent decompressive craniectomy, resulting in coma. The times from onset of symptoms to establishment of CPB were 192 minutes and 416 minutes, and times to circulatory arrest in the brain were 28 minutes and 36 minutes, respectively. The $\mathrm{rSO}_{2}$ in near-infrared spectroscopy increased after starting $\mathrm{CPB}$ in these patients, and increased blood flow in the true lumen of the BCA was identified on intraoperative echocardiography. Although the postoperative CT scans showed severe cerebral edema, postoperative magnetic resonance imaging and $\mathrm{CT}$ scan with 3-dimensional reconstruction showed patent intracranial and cervical arteries (Figure 6). Furthermore, the first patient required a large volume of perioperative infusion for shock, and the second patient underwent a prolonged time before restoration of cerebral perfusion. These results suggest the possibility that cerebral edema may have developed as the result of cerebral reperfusion injury in these patients. Because postoperative cerebral edema was not found in other patients, this condition was identified as a poor prognostic factor.

\section{Study Limitations}

This study has several limitations. First, the sample size was small. Second, a comparative study using other blood supply procedures was not performed. Third, all patients underwent emergency surgery, and near-infrared spectroscopy was not performed in some cases because of limitations of staff and facilities.

\section{CONCLUSIONS}

EGACS has potential as a rapid and reliable route of antegrade central systemic perfusion during surgery for TAAAD complicated by stroke due to CM. However, further quantitative analysis of the blood flow in the dissected aorta is warranted for this method.

\section{Conflict of Interest Statement}

Authors have nothing to disclose with regard to commercial support.
The authors thank Editage (www.editage.jp) for English language editing.

\section{References}

1. Bossone E, Corteville DC, Harris KM, Suzuki T, Fattori R, Hutchison S, et al. Stroke and outcomes in patients with acute type A aortic dissection. Circulation. 2013;128:S175-9.

2. Czerny M, Schoenhoff F, Etz C, Englberger L, Khaladj N, Zierer A, et al. The impact of pre-operative malperfusion on outcome in acute type A aortic dissection: results from the GERAADA registry. J Am Coll Cardiol. 2015;65: 2628-35.

3. Tsukube T, Hayashi T, Kawahira T, Haraguchi T, Matsukawa R, Kozawa S, et al. Neurological outcomes after immediate aortic repair for acute type A aortic dissection complicated by coma. Circulation. 2011;124:S163-7.

4. Estrera AL, Garami Z, Miller CC, Porat EE, Achouh PE, Dhareshwar J, et al. Acute type A aortic dissection complicated by stroke: can immediate repair be performed safely? J Thorac Cardiovasc Surg. 2006;132:1404-8.

5. Pocar M, Passolunghi D, Moneta A, Mattioli R, Donatelli F. Coma might not preclude emergency surgery in acute aortic dissection. Ann Thorac Surg. 2006; 81:1348-51.

6. Frederick JR, Yang E, Trubelja A, Desai ND, Szeto WY, Pochettino A, et al. Ascending aortic cannulation in acute type A dissection repair. Ann Thorac Surg. 2013;95:1808-11.

7. Inoue Y, Takahashi R, Ueda T, Yozu R. Synchronized epiaortic two-dimensional and color Doppler echocardiographic guidance enables routine ascending aortic cannulation in type A acute aortic dissection. J Thorac Cardiovasc Surg. 2011; 141:354-60.

8. van Swieten JC, Koudstaal PJ, Visser MC, Schouten HJ, van Gijn J. Interobserver agreement for the assessment of handicap in stroke patients. Stroke. 1988;19: 604-7.

9. Frese E, Brown M, Norton B. Clinical reliability of manual muscle testing: middle trapezius and gluteus medius muscles. Phys Ther. 1987;67:1072-6.

10. Watanabe K, Tanaka M, Yuki S, Hirai M, Yamamoto Y. How is edaravone effective against acute ischemic stroke and amyotrophic lateral sclerosis? J Clin Biochem Nutr. 2018;62:20-38.

11. Masuda M, Okumura M, Doki Y, Endo S, Hirata Y, Kobayashi J, et al. Thoracic and cardiovascular surgery in Japan during 2014: annual report by the Japanese Association for Thoracic Surgery. Gen Thorac Cardiovasc Surg. 2016;64: 665-97.

12. Orihashi K. Cerebral malperfusion in acute aortic dissection. Surg Today. 2016; 46:1353-61.

13. Di Eusanio M, Patel HJ, Nienaber CA, Montgomery DM, Korach A, Sundt TM, et al. Patients with type A acute aortic dissection presenting with major brain injury: should we operate on them? J Thorac Cardiovasc Surg. 2013;145:S213-21.

14. Bonser RS, Ranasinghe AM, Loubani M, Evans JD, Thalji NM, Bachet JE, et al. Evidence, lack of evidence, controversy, and debate in the provision and performance of the surgery of acute type A aortic dissection. J Am Coll Cardiol. 2011:58:2455-74.

15. Hagan PG, Nienaber CA, Isselbacher EM, Bruckman D, Karavite DJ, Russman PL, et al. The international registry of acute aortic dissection (IRAD): new insights into an old disease. JAMA. 2000;283:897-903.

16. Cambria RP, Brewster DC, Gertler J, Moncure AC, Gusberg R, Tilson MD, et al. Vascular complications associated with spontaneous aortic dissection. J Vasc Surg. 1988;7:199-209.

17. Ergin MA, Galla JD, Lansman S, Griepp RB. Acute dissections of the aorta. Current surgical treatment. Surg Clin North Am. 1985;65:721-41.

18. Picconne W Jr, Hamilton IN, Najafi H. Intentional delayed repair of acute dissection of the ascending aorta complicated by stroke. J Thorac Cardiovasc Surg. 1995; 109:807-8.

19. Tsukube T, Haraguchi T, Okada Y, Matsukawa R, Kozawa S, Ogawa K, et al. Long-term outcomes after immediate aortic repair for acute type A aortic dissection complicated by coma. J Thorac Cardiovasc Surg. 2014; 148:1013-9.

20. Astrup J, Siesjö BK, Symon L. Thresholds in cerebral ischemia - the ischemic penumbra. Stroke. 1981;12:723-5.

21. Powers WJ, Derdeyn CP, Biller J, Coffey CS, Hoh BL, Jauch EC, et al. 2015 American Heart Association/American Stroke Association focused update of the 2013 guidelines for the early management of patients with acute ischemic stroke regarding endovascular treatment. A guideline for healthcare professionals from the American Heart Association/American Stroke Association. Stroke. 2015;46:3020-35. 
22. Abe T, Usui A. The cannulation strategy in surgery for acute type A dissection. Gen Thorac Cardiovasc Surg. 2017;65:1-9.

23. Rylski B, Urbanski PP, Siepe M, Beyersdorf F, Bachet J, Gleason TG, et al. Operative techniques in patients with type A dissection complicated by cerebral malperfusion. Eur J Cardiothorac Surg. 2014;46:156-66.

24. Ren Z, Wang Z, Hu R, Wu H, Deng H, Zhou Z, et al. Which cannulation (axillary cannulation or femoral cannulation) is better for acute type A aortic dissection repair? A meta-analysis of nine clinical studies. Eur J Cardiothorac Surg. 2015;47:408-15.

25. Benedetto U, Mohamed H, Vitulli P, Petrou M. Axillary versus femoral arterial cannulation in type A acute aortic dissection: evidence from a meta-analysis of comparative studies and adjusted risk estimates. Eur J Cardiothorac Surg. 2015;48:953-9.

26. Khaladj N, Shrestha M, Peterss S, Strueber M, Karck M, Pichlmaier M, et al. Ascending aortic cannulation in acute aortic dissection type A: the Hannover experience. Eur J Cardiothorac Surg. 2008;34:792-6.

27. Inoue Y, Ueda T, Taguchi S, Kashima I, Koizumi K, Takahashi R, et al. Ascending aorta cannulation in acute type A aortic dissection. Eur J Cardiothorac Surg. 2007;31:976-9.

28. Sabashnikov A, Heinen S, Deppe AC, Zeriouh M, Weymann A, Slottosch I, et al. Axillar or aortic cannulation for aortic repair in patients with Stanford A dissection? Ann Thorac Surg. 2016;102:787-94.
29. Kamiya H, Kallenbach K, Halmer D, Ozsoz M, Ilg K, Lichtenberg A, et al Comparison of ascending aorta versus femoral artery cannulation for acute aortic dissection type A. Circulation. 2009;120:S282-6.

30. Orihashi K, Sueda T, Okada K, Takahashi S. Compressed true lumen in the innominate artery: a pitfall of right axillary arterial perfusion in acute aortic dissection. J Thorac Cardiovasc Surg. 2009;137:242-3.

31. Igarashi T, Takahashi S, Takase S, Yokoyama H. Intraoperative thrombectomy for occluded carotid arteries in patients with acute aortic dissection: report of two cases. Surg Today. 2014;44:1177-9.

32. Urbanski PP. Carotid artery cannulation in acute aortic dissection with malperfusion. J Thorac Cardiovasc Surg. 2006;131:1398-9.

33. Sakaguchi G, Komiya T, Tamura N, Obata S, Masuyama S, Kimura C, et al Cerebral malperfusion in acute type A dissection: direct innominate artery cannulation. J Thorac Cardiovasc Surg. 2005;129:1190-1.

34. Okita Y, Matsumori M, Kano H. Direct reperfusion of the right common carotic artery prior to cardiopulmonary bypass in patients with brain malperfusion complicated with acute aortic dissection. Eur J Cardiothorac Surg. 2016;49:1282-4.

Key Words: ascending aortic central cannulation, cerebral malperfusion, coma, EGACS, stroke, type A acute aortic dissection 